\title{
Profile of Low Vision Patients in a Resource-Poor Underserved Setting of a Developing Country
}

\author{
C. C. Eze1, O. I. Okoye ${ }^{2}$, O. Okoye ${ }^{2 *}$, N. Nwachukwu², N. N. Okoloagu ${ }^{1}$, \\ E. N. Onwasigwe ${ }^{2}$ \\ ${ }^{1}$ Department of Ophthalmology, Enugu State University Teaching Hospital Parklane, Enugu, Nigeria \\ ${ }^{2}$ Department of Ophthalmology, University of Nigeria Teaching Hospital, Ituku Ozalla, Enugu, Nigeria \\ Email:*eagleobi@yahoo.com
}

How to cite this paper: Eze, C.C., Okoye, O.I., Okoye, O., Nwachukwu, N., Okoloagu, N.N. and Onwasigwe, E.N. (2018) Profile of Low Vision Patients in a Resource-Poor Underserved Setting of a Developing Country. Open Journal of Ophthalmology, 8, 120-131.

https://doi.org/10.4236/ojoph.2018.82016

Received: April 16, 2018

Accepted: May 18, 2018

Published: May 21, 2018

Copyright (c) 2018 by authors and Scientific Research Publishing Inc. This work is licensed under the Creative Commons Attribution-NonCommercial International License (CC BY-NC 4.0). http://creativecommons.org/licenses/by-nc/4.0/

\begin{abstract}
Objectives: To identify causes of low vision among the patients in the Eye clinic of University of Nigeria Teaching Hospital (UNTH). Methods: This was a cross-sectional study of all new consecutive low vision patients seen at the eye clinic of University of Nigeria Teaching Hospital-(UNTH) Ituku-Ozalla. All patients with low vision were evaluated by the researcher and the findings entered on a research protocol. Data were analyzed using descriptive statistics to generate frequency and percentage distributions and analytical statistics to test for significance of observed inter-group differences. In all comparisons, statistical significance was indicated by $\mathrm{p}<0.05$. Results: A total of 197 patients were seen comprising of 120 males and 77 females (M:F $=1.6: 1)$ aged $39.3 \pm 22.9 \mathrm{SD}$ years. The main causes of low vision in the study population were glaucoma (36.0\%), followed by oculocutaneous albinism (14.7\%). The mean presenting distant VA was logMAR 1.1 (95\% CI), while the mean near VA is logMar $1.0(6 / 60,20 / 200)$. After refraction, the mean distant VA was logMar 1.0, using unpaired t-test, the difference between the distant presenting and refracted VA were not statistically significant. 57.9\% had distant VA after optical low vision assessment of logMar 0.9 - logMar 0.2 while the mean distant VA was logMar 0.8 which was statistically significant. Near vision after optical assessment improved with a mean of logMar 0.8 , which was statistically significant. Conclusion: Glaucoma was the commonest cause of low vision in this study. Optical low vision aids improved the visual functions of majority of the patients in this study.
\end{abstract}

\section{Keywords}

Low Vision, Profile, Nigeria 


\section{Introduction}

Over 285 million people in the world are visually impaired, of whom 39 million are blind and 246 million have low vision. In Africa, 20.4 million people have low vision which is equal to the number 25.4 thousand/million population [1].

About $90.0 \%$ of the world visually impaired live in developing countries [1]. This places a lot of burden on the developing countries including sub-Saharan Africa that have scarce resources. On a global scale, low vision is a major public health issue, especially due to the increased burden of the ageing population [2]. About $65.0 \%$ of all people who are visually impaired are aged 50 years and older [2]. Globally, 58 million people turn 60 years annually, and by 2050 the 60 -plus cohort will reach two billion [2].

Equally, low vision is a major cause of morbidity and has profound effects on quality of life. They inhibit mobility and economic well-being of the individuals affected, as well as their families [3]. Nevertheless, low vision services have suffered from neglect in organized eye care, especially in low-income countries [3].

In the African continent, which is mainly a low income continent, the burden of low vision is high for those affected [1].

Based on figures from the Nigeria National Blindness and Visual impairment survey, it is estimated that approximately 800,000 individuals have functional low vision in Nigeria [4].

Functionally, low vision is characterized by irreversible visual loss and a reduced ability to perform many daily activities [5]. It is an important public health problem [6]; and provision of low vision services is one of the priorities in the global initiative, VISION 2020-The Right to Sight [7], and also in achieving Universal eye health: a global action plan (GAP) 2014-2019 [8].

There are approximately 5000 adults/million populations in Nigeria who require low vision assessment [8]. The challenge of providing low vision services for such a large population is enormous and requires efficient use of available resources.

Majority of available data on people with low vision is derived from population based surveys which were not specifically designed to study functional low vision. The clinical studies which give more detailed information on patients actually attending low vision clinics are few [9] for example; Ezepue's prevalence study on Magnitude and causes of blindness and low vision in Anambra State did not provide clinical details of low vision patients [10]. It is therefore important to collect and analyze clinical and demographic data from patients with low vision in order to deliver appropriate low vision care [9]. Findings will inform policy formulation and implementation by eye care stakeholders that will in turn engender efficient and cost effective low vision services to those that need it in South East Nigeria and elsewhere.

\section{Material and Methods}

\subsection{Study Area}

UNTH is located 21 kilometres from Enugu Capital City along Enugu-Port 
Harcourt Express way. Services rendered by university of Nigeria Teaching Hospital extend to various states in this country particularly those in the south-East, South-South and North-Central Geo-political zones.

\subsection{Study Design and Scope}

This was a cross-sectional study of all consecutive new low vision patients seen at the low vision unit of the eye clinic of University of Nigeria Teaching Hospital, Ituku-Ozalla between November 2014 and November 2015. Patients included were consenting patients who presented at the eye clinic having been treated at the main eye clinic for various ailments but whose visual needs were not adequately met by conventional methods in accordance with the Bangkok definition of low vision [11]. Thus, most subjects with operable cataracts were not routinely referred for low vision assessment and were not included in this study.

A person with low vision is one who has impaired visual function despite treatment of eye disease and/or correction of refractive error, and has reduced visual acuity in the better eye which is less than $6 / 18$ but better than light perception (LP) or a visual field constriction to less than $10^{\circ}$, but who uses or is potentially able to use vision for the planning and/ or execution of a task [11]. This definition of low vision excludes individuals whose visual acuity could be improved by surgical and/or medical treatment.

\subsection{Sample Size and Sample Procedure}

The calculated minimum sample size of 179 was based on a $3 \%$ prevalence rate of low vision in a previous hospital based survey [12], 95\% confidence interval and a $5 \%$ margin of error. The calculated minimum sample size was inflated to a modified sample of 197 to achieve wider coverage.

\subsection{Ethical Considerations}

Prior to the commencement of the study, Ethics approval consistent with the tenets of 1964 Helsinki declaration on research involving human subjects was obtained from UNTH's Medical and Health Research Ethics Committee (Institutional Review Board). A written informed consent was obtained from each participant.

\subsection{Study Procedure}

All patients with low vision were seen by the researcher and the findings entered on a research protocol.

Section A of the protocol consists of Socio-demographic data regarding age, gender, marital status, education, employment status and area of residency.

Section B of the protocol consists of main presenting history, past ophthalmic history, and associated visual symptoms, functional visual problems, psychological challenges and general health condition.

Section C consists of ocular examinations which included: presenting distance 
and near visual acuities, distance vision was assessed with a pin hole to see if there was improvement, following which the patient with improvement was refracted with Welch Allyn retinoscope and subjective refraction to get the best corrected visual acuities for near and distance. Visual acuity (VA) for distance was assessed with the use of Low Vision Resource Centre (LVRC) Bailey-Lovie Sloan Letters design charts and recorded in logarithm of the minimum angle of resolution (log MAR) unit at the standard test distance of 4 meters. Near visual acuity was recorded with LVRC near acuity chart at a distance of $45 \mathrm{~cm}$. LVRC tumbling E chart was used for adults that are illiterates. Good-Lite Lea symbols with testing distance of 3 meters were used for young children; older children were tested with LVRC charts for distance and near vision. Each eye was assessed separately. Inability to identify letters or symbols was followed by attempts to get the visual acuity by reducing the distance between the patient and the charts, and also by counting fingers, hand movement and light perception. Color vision was tested with Bright Colours 12 Pencils Contrast sensitivity was assessed with Good-Lite Hiding Heidi low contrast flip chart.

Anterior segment examination was done with a pen torch and slit lamp biomicroscope (Haag-Streit). Pupillary reaction was assessed with a pen torch. Direct fundoscopy with Welch-Allyn (model 18,200) and indirect ophthalmoscopy with +20 dioptres. The pupils were dilated with $0.5 \%$ tropicamide and phenylephrine combination (Generic name Trophen) when necessary. Central visual field analysis was done were applicable with Humphrey Standard Automated Perimetry (SAP). Intraocular pressure (IOP) was measured where necessary with Goldmann applanation tonometry.

Section D consists of the clinical diagnosis/cause of low vision and interventions recommended which included objective refraction with Welch-Allyn streak retinoscope and subjective refractions. A trial of telescopes was done and the visual acuities with the telescopes noted. Trial of magnifiers for near vision was done and the visual acuity noted. Optical low vision aids was prescribed according to the patients' preferences.

Non optical low vision aids used were typoscopes for writing guide, face caps to reduce glare, goose necked lamp stands to improve illumination for patients while reading. Black pens for writing on white papers in order to improve contrast. Patients were helped to easily access low vision devices and was helped to acquire visual training and rehabilitation.

\subsection{Data Management}

Data on each participant was carefully cleaned, coded and double entries were made into the computer. Analysis of the data was done with the Statistical Package for Social Sciences VERSION 18 (SPSS Inc., Chicago, Illinois, USA). The statistical tools that were used for data analysis included chi-square tests which measured association between two quantitative variables. Student t-test was used for continuous variables. Multiple regression analysis was done for multiple variables. Data presentations were with tables, charts and in prose. For all compar- 
isons, a P-Value of $<0.05$ at one degree of freedom was considered significant.

\section{Results}

A total of 197 patients took part in the study comprising of 120 (60.9\%) males and 77 (39.1\%) female (M:F ratio 1.6:1) aged $39.3 \pm 22.9$ SD (range 6 to 91 years). One hundred and eighty eight (95.4\%) had at least primary education (Table 1).

Seventy seven patients (39.1\%) had presenting visual acuity (VA) in the better eye of less than counting fingers at 4 meters (>logMar 1.3, >20/400, >6/120), Table 2. The presenting near VA ranges from $>\log$ Mar 1.3 (CF, HM, PL) to log 0.0 $(20 / 20,6 / 6), 72(36.5 \%)$ had VA of $>\log$ Mar $1.3,62.4 \%$ of the patients had a presenting near VA > logMar 1.0 (Table 2). After refraction, the mean distant VA was logMar 1.0, using unpaired t-test, the difference between the distant presenting and refracted VA were not statistically significant.

Results from the Table 3 below, showed that $57.9 \%$ had distant VA after optical low vision assessment of $\log$ Mar 0.9 (20/160, 6/48) - logMar - 0.2 (20/12.5, 6/4). The mean distant VA were logMar 0.8 and this was statistically significant when compared with the mean value of both the presenting and refraction $\mathrm{VA}$. Most $(84.1 \%)$ of those with distant VA of $>\log$ Mar 1.3 did not have any improvement after low vision assessment, 75.9\% of those with VA of logMar 1.0 1.3 had an average improvement of 7 lines to logMar 0.5 majority (75.0\%) of those with VA of log Mar 0.9 - 0.5 had an average improvement of 3 lines to log 0.4 .

Near vision after optical assessment improved with a mean of logMar 0.8, which was statistically significant when compared with the mean presenting near VA (Table 3). Furthermore, $91.1 \%$ of those with near VA of $>\log$ Mar 1.3 did not have any improvement for near vision, while $8.9 \%$ had an average improvementto logMar 0.9. In addition, $71.4 \%$ of those with near VA between logMar $0.4-0.1$ had an average improvement of 2 lines with final VA of logMar 0.0.

Only $84(42.6 \%)$ of the patients have been previously diagnosed with eye diseases prior to presentation. Almost all the respondents believed that they are in general good health, but 15 (7.6\%) have hypertension and 11 (5.6\%) have diabetes, while only 3 (1.5\%) have hand tremors.

Diminished vision was the main presenting complaints of most $(90.9 \%)$ of the participants followed by reduction in field of view (49.7\%). The mean duration of presenting complaints was $11.7 \pm 10.1$ SD years. The visual loss were gradually progressive in 151 (76.6\%) patients while it was sudden progressive in 15 (7.6\%) of the patients. It were non progressive in $27(13.7 \%)$ of the patients. The number of participants that used glasses for vision was 105 (53.3\%). The causes of low vision in the study population were glaucoma (36.0\%) followed by oculo-cutaneous albinism (14.7\%) and amblyopia (8.1\%) (Table 4).

From the study, 114 (57.9\%) of the patients would benefit from optical low vision aid while $83(42.1 \%)$ would benefit from non-optical low vision aids after 
C. C. Eze et al.

Table 1. Socio-Demographic characteristics of 197 participants at UNTH low vision clinic.

\begin{tabular}{|c|c|c|c|c|}
\hline Age (years) $\mathrm{N}=197$ & $\mathrm{n}$ & M & $\mathrm{F}$ & $\mathrm{n} \%$ of $\mathrm{N}$ \\
\hline $5-15$ & 32.0 & 18.0 & 14.0 & 16.2 \\
\hline $16-25$ & 45.0 & 29.0 & 16.0 & 22.8 \\
\hline $26-35$ & 24.0 & 12.0 & 12.0 & 12.2 \\
\hline $36-45$ & 24.0 & 15.0 & 9.0 & 12.2 \\
\hline $46-55$ & 22.0 & 16.0 & 6.0 & 11.2 \\
\hline $56-65$ & 15.0 & 12.0 & 3.0 & 7.6 \\
\hline $66-75$ & 18.0 & 8.0 & 10.0 & 9.1 \\
\hline $76-85$ & 15.0 & 8.0 & 7.0 & 7.6 \\
\hline $86-100$ & 2.0 & 2.0 & 0.0 & 1.0 \\
\hline total & 197.0 & 120.0 & 77.0 & 100.0 \\
\hline Characteristics & \multicolumn{2}{|c|}{$\mathrm{N}=197$} & \multicolumn{2}{|c|}{$\mathrm{N}=197$} \\
\hline \multicolumn{5}{|l|}{ educational level } \\
\hline primary & \multicolumn{2}{|c|}{92.0} & \multicolumn{2}{|c|}{46.7} \\
\hline commercial & \multicolumn{2}{|c|}{2.0} & \multicolumn{2}{|c|}{1.0} \\
\hline secondary & \multicolumn{2}{|c|}{53.0} & \multicolumn{2}{|c|}{26.9} \\
\hline tertiary & \multicolumn{2}{|c|}{41.0} & \multicolumn{2}{|c|}{20.8} \\
\hline none & \multicolumn{2}{|c|}{9.0} & \multicolumn{2}{|c|}{4.6} \\
\hline \multicolumn{5}{|l|}{ Marital status } \\
\hline single & \multicolumn{2}{|c|}{101.0} & \multicolumn{2}{|c|}{51.3} \\
\hline married & \multicolumn{2}{|c|}{80.0} & \multicolumn{2}{|c|}{40.6} \\
\hline widowed & \multicolumn{2}{|c|}{14.0} & \multicolumn{2}{|c|}{7.1} \\
\hline divorced/separated & \multicolumn{2}{|c|}{2.0} & \multicolumn{2}{|c|}{1.0} \\
\hline location & \multicolumn{4}{|c|}{ of residency } \\
\hline urban & \multicolumn{2}{|c|}{110.0} & \multicolumn{2}{|c|}{55.8} \\
\hline rural & \multicolumn{2}{|c|}{87.0} & \multicolumn{2}{|c|}{44.2} \\
\hline \multicolumn{5}{|l|}{ occupation } \\
\hline student & \multicolumn{2}{|c|}{61} & \multicolumn{2}{|c|}{31} \\
\hline artisan & & & & \\
\hline retired & & & & \\
\hline trader & & & & \\
\hline unemployed & & & & \\
\hline others & & & & \\
\hline
\end{tabular}

${ }^{\star}$ House wives, partly employed, unable to work, etc.

assessment of the patients with optical low vision devices. In the group, that had improvement with optical devices, $38.2 \%$ required only telescopes, $14.5 \%$ 
Table 2. Presenting VA in the better eye for 197 low vision patients at eye clinic UNTH.

\begin{tabular}{ccc}
\hline Presenting VA $(\log M A R)$ & $\mathrm{N}=197$ & $\mathrm{n} \%=100$ \\
\hline Distance & 77.0 & 39.1 \\
\hline$>1.3$ & 59.0 & 29.9 \\
$1.3-1.0$ & 52.0 & 26.4 \\
$0.9-0.5$ & 0.0 & 0.0 \\
$0.4-0.1$ & 9.0 & 4.6 \\
$0.0--0.2$ & & \\
Near & 72 & 36.5 \\
$>1.3$ & 51 & 25.9 \\
$1.3-1.0$ & 49 & 24.9 \\
$0.9-0.5$ & 13 & 6.6 \\
$0.4-0.1$ & 12 & 6.1 \\
\hline $0.0--0.2$ & & \\
\hline
\end{tabular}

Key: logMAR means logarithm of minimum angle of resolution.

Table 3. VA after assessment with optical low vision aids of the study population at UNTH.

\begin{tabular}{ccc}
\hline VA (logMAR) & $\mathrm{N}=197$, & $\mathrm{N} \%=100$ \\
\hline Distant & 65.0 & 33.0 \\
\hline 1.3 & 18.0 & 9.1 \\
$1.3-1.0$ & 49.0 & 24.9 \\
$0.9-0.5$ & 36.0 & 18.3 \\
$0.4-0.1$ & 29.0 & 14.7 \\
$0.0--0.2$ & & \\
Near & 66.0 & 33.5 \\
$>1.3$ & 22.0 & 11.2 \\
$1.3-1.0$ & 28.0 & 14.2 \\
$0.9-0.5$ & 66.0 & 33.5 \\
$0.4-0.1$ & 15.0 & 7.6 \\
\hline $0.0-0.2$ & & \\
\hline
\end{tabular}

required only magnifiers, $32.8 \%$ required both telescopes and magnifiers while $14.5 \%$ required both optical and non-optical devices like face caps, antiglare glasses, table lamps etc.

An analysis of optical improvement based on aetiology in this study showed that albinism had the most (93.1\%) optical improvement in vision, followed by ARMD (73.3\%). The power of prescribed optical aids ranges from $3 \times$ to $9 \times$.

Colour perception were abnormal in 88 (44.7\%) of the study population and all the patients with abnormal colour perception had presenting visual acuity of $\log 0.9(20 / 160,6 / 48)$ or less. Majority of the participants, $165(83.6 \%)$ have no 
Table 4. Causes of low vision among the 197 patients.

\begin{tabular}{ccc}
\hline Diseases & frequency & N\% \\
\hline Glaucoma & 71.0 & 36.0 \\
Albinism & 29.0 & 14.7 \\
Amblyopia & 16.0 & 8.1 \\
Age-Related Macular Degeneration & 15.0 & 7.6 \\
Optic atrophy & 13.0 & 6.7 \\
Patholological myopia & 7.0 & 3.6 \\
Retinitis pigmentosa & 7.0 & 3.6 \\
Cornea opacity & 7.0 & 3.6 \\
Retinal detachment & 7.0 & 3.6 \\
Maculopathies & 5.0 & 2.5 \\
Toxoplasmosis & 5.0 & 2.5 \\
Micropthalmos & 4.0 & 2.0 \\
Chronic uveitis & 2.0 & 1.0 \\
Hypolpastic optic disc & 1.0 & 0.5 \\
Congenital aniridia & 1.0 & 0.5 \\
Diabetic retinopathy & 1.0 & 0.5 \\
Complicated ICCE & 1.0 & 0.5 \\
Unknown & 5.0 & 2.5 \\
total & 197.0 & 100.0 \\
\hline
\end{tabular}

Key: ICCE means Intracapsular cataract extraction.

low vision training while $32(16.2 \%)$ have low vision training. Among those with no previous low vision training, $75.4 \%$ will like to go for low vision training while $24.6 \%$ will not. Many of the patients $(89.8 \%$ ) have not used any low vision aid.

\section{Discussion}

There were more males $(\mathrm{M})$ than females $(\mathrm{F})$ in this study in all the age groups. Various studies on gender distribution of low vision patients presenting to the hospital have reported significant male preponderance [13] [14] [15], and female preponderance [16] [17]. The observed discrepancies are likely due to socioeconomic and cultural differences between study areas/settings. In low and medium income countries (LMICs), the prevailing socioeconomic settings characterized by unhindered male access to family finance and extension healthcare may account for this trend.

The mean age of the participants in this study is similar to that to the 48.0 years observed by Richard et al. [14] in Bayelsa Nigeria and 43.3 years in Otulana's [18] Ogun Nigerian cohort. This is contrast to findings in developed settings where Goldstein et al. [16] in USA observed a mean age of 77.0 years and Ikesugi et al. [19] 70.6 years in Japan. 
This difference in the pattern of the age distribution may be a reflection of the older ageing populations in developed countries [9].

Across all age groups glaucoma accounted for the commonest cause of low vision in this study followed by oculocutaneous albinism. This agrees with the findings in the Nigerian national blindness and visual impairment survey in which glaucoma was the most common cause of low vision [4]. Similarly, Ikesugi et al. [19] reported glaucoma as the leading cause of low vision in their cohort. Globally, glaucoma remains the second leading cause of blindness and the leading cause of irreversible blindness [20]. This underscores the importance of early diagnosis and treatment of glaucoma to reduce this trend. Globally, several surveys has reported the most common cause of low vision as retinitis pigmentosa [9], cataract [15], and age related macular degeneration [20] [21] [22] [23]. A number of factors may account for these observed discrepancies such as possible poor uptake of low vision services by patients with glaucoma in the study area, poor referral of glaucoma patients with low vision to the low vision clinic and higher number of aging population with the attendant higher prevalence of age-related macular degeneration as seen in the developed setting.

The most common cause of low vision in children aged $5-15$ years in this study were amblyopia followed by oculocutaneous albinism and cornea opacity. This is similar to findings in other surveys [24] [25] while other surveys [9] [26] reported albinism, retinitis pigmentosa and hereditary macular disease as common causes of low vision in children. Differences in study areas and setting may largely account for this. The findings of retina hereditary disorders as common causes were largely observed in areas of high consanguinity marriages. Genetic counseling and cultural changes is suggested as a way to reduce this trend.

Majority of the patients in this study were able to achieve improvement in VA (both distance and near) after low vision assessment. In a study in Ontario, Canada on the effectiveness of a low vision clinic, the researchers found that benefits from attending the clinic were reported by $89.5 \%$ of patients and $81.0 \%$ of patients were regularly using low vision aids [27]. This contrast with this present study where $57.9 \%$ could benefit from optical low vision aids. The difference from this study was related to the severity of presenting VA in this study where $69 \%$ had VA $\geq \log$ MAR 1.0. These groups of patients had the least improvement in VA after optical low vision assessment.

A study in Britain shows that approximately $85.0 \%$ of those known to be visually impaired had useful residual vision and could benefit from visual rehabilitation [28]. There was good evidence that prescribed low vision devices are used and valued by the users. Furthermore, a study by Reeves et al comparing different types of low vision service provision in the UK for people with AMD found that at 4 and 12 months, $95 \%$ and $94 \%$ respectively of participants reported using at least one low vision device [29]. Thus a high prevalence of patients with low vision can actually benefit from low vision treatment. Equally, studies done by van Rens et al in the Netherlands and Temel, in Turkey, found that low vision devices were prescribed for $79.7 \%$ [30] and 77\% [31] of the total participants re- 
spectively. But this high percentage of prescribed low vision compared to this study could be because the participants were in the developed countries and could have presented earlier for low vision assessment.

But in a study in India [26], all the patients seen were able to achieve a distant VA of log Mar 0.5 (6/18) and near VA of at least N10 (logMar 0.5). This is in contrast from this study. The difference could be related to the initial presenting VA; in this present study, more than $60.0 \%$ had VA of $>\log 1.0$ while in the Indian study, only $29.9 \%$ had VA $>\log 1.0$. Worse presenting VA is associated with less chance of optical improvement with low vision aids.

\section{Limitations of the Study}

Information obtained from this study was clinic-specific and may be strongly influenced by the sources of referral to low vision clinic in UNTH. Furthermore, acceptance and utilisation of low vision services by the population served by the hospital could have influenced the outcome of this study. In addition, the sample size of this study was limited when compared to population surveys. Thus, it may be prone to sampling errors and it could be limited in extrapolation to the general population.

\section{Conclusion}

There were more males than females in the study. Glaucoma was the commonest cause of low vision in this study. But for the age group 5 - 15 years, amblyopia was the commonest cause of low vision. Optical low vision aids improved the visual functions of majority of the patients in this study. There is a need for integration of low vision services into the national eye health plan. This will help the country to achieve the Vision 2020 and the sustainable development goals.

\section{References}

[1] WHO (2014) Visual Impairment and Blindness. Fact Sheet $\mathrm{N}^{\circ} 282$. http://www.who.int/mediacentre/factsheets/fs282/en/

[2] Braun, J. (2014) Dependency Costs of Blindness and Low Vision in Older Adults; Literature Review. Splaine Consulting 2012. http://www.ifa.fiv.org

[3] Safal, K. and Pekila, L. (2013) Profile of Low Vision Population Attending Low Vision Clinic in a Peripheral Eye Hospital in Nepal. Optometry \& Visual Performance, 1, 209-213.

[4] Entekume, G., Patel, J., Sivasubramaniam, S., Gilbert, C., Ezelum, C., Murthy, G.V. and Rabiu, M. (2011) Prevalence, Causes and Risk Factors for Functional Low Vision in Nigeria: Results from the National. Investigative Ophthalmology \& Visual Science, 52, 6714-6719. https://doi.org/10.1167/iovs.11-7293

[5] Van-Dijk, K. (2007) Providing Care for Children with Low Vision. Community Eye Health, 20, 24-25.

[6] Kim, J.H., Joo, K.S. and Moon, N.J. (2010) Characteristics of 681 Low Vision Patients in Korea. Journal of Korean Medical Science, 25, 1217-1221. https://doi.org/10.3346/jkms.2010.25.8.1217

[7] World Health Organisation (1997) Global Initiative for the Elimination of Avoida- 
ble Blindness. WHO/PBL/97.61_Rev.1, Geneva.

[8] WHO (2014) Universal Eye Health: A Global Action Plan, 2014-2019.

http://www.who.int/blindness/en/

[9] Olusanya, B., Godfrey, O., Weheed, I. and Bekibele, C. (2012) Profile of Patients Presenting at a Low Vision Clinic in a Developing Country. BMC Ophthalmology, 12, 31. https://doi.org/10.1186/1471-2415-12-31

[10] Ezepue, U.F. (1997) Magnitude and Causes of Blindness and Low Vision in Anambra State of Nigeria (Results of 1992 Point Prevalence Survey). Journal of Public Health, 111, 305-309. https://doi.org/10.1016/S0033-3506(97)00059-0

[11] World Health Organization (1993) The Management of Low Vision in Children. Report of a WHO Consultation: Bangkok, July 23-24 1992. WHO (WHO/PBL/93.27), Geneva.

[12] Khan, S.A. (2000) A Retrospective Study of Low-Vision Cases in an Indian Tertiary Eye-Care Hospital. Indian Journal of Ophthalmology, 48, 201.

[13] Nasiru, M., Rabiu, M.M., Adamu, M.D., Elizabeth, E. and Isiyaku, S. (2011) Prevalence and Causes of Blindness and Visual Impairment in Sokoto State, Nigeria: Baseline Data for Vision 2020: The Right to Sight Eye Care Programme. Middle East African Journal of Ophthalmology, 18, 123-128. https://doi.org/10.4103/0974-9233.80700

[14] Richard, A.I. (2010) Causes of Blindness and Low Vision in Bayelsa State, Nigeria: A Clinic Based Study. Nigerian Quarterly Journal of Hospital Medicine, 20, 125-128.

[15] Melese, M., Alemayehu, W., Bayu, S., Girma, T., Hailesellasie, T., Khandekar, R., Worku, A. and Courtright, P. (2003) Low Vision and Blindness in Adults in Gurage Zone, Central Ethiopia. British Journal of Ophthalmology, 87, 677-680. https://doi.org/10.1136/bjo.87.6.677

[16] Goldstein, J.E., Massof, R.W., Deremeik, J.T., Braudway, S., Jackson, M.L., Kehler, K.B., Primo, S.A. and Sunness, J.S. (2012) Baseline Traits of Low Vision Patients Served by Private Outpatient Clinical Centres in the United States. Archives of Ophthalmology, 130, 1028-1037. https://doi.org/10.1001/archophthalmol.2012.1197

[17] Maberley, D.A., Hollands, H., Chuo, J., Tam, G., Konkal, J., Roesch, M., Veselinovic, A., Witzigmann, M. and Bassett, K. (2006) The Prevalence of Low Vision and Blindness in Canada. Eye, 20, 341-346. https://doi.org/10.1038/sj.eye.6701879

[18] Otulana, T.O. (2012) Blindness and Visual Impairment in Remo, Ogun State: A Hospital-Based Study. Nigerian Postgraduate Medical Journal, 19, 153-156.

[19] Ikesugi, K., Tsukitome, H., Yagi, T., Yamaguchi, T., Tamei, I., Sasoh, M. and Uji, Y. (2010) Causes of Visual Impairment in Mie Prefecture during a 5-Year Period. Journal of Japanese Ophthalmological Society, 114, 505-511.

[20] World Health Organization (2004) Glaucoma Is Second Leading Cause of Blindness. Bulletin of the World Health Organization, 82, 811-890.

[21] Owsley, C., McGwin, G. and Searcey, K. (2009) Characteristics of Low Vision Rehabilitation Services in the United States. Archives of Ophthalmology, 127, 681-689.

[22] Maberley, D.A., Hollands, H., Chang, A., Adilman, S., Chakraborti, B. and Kliever, G. (2007) The Prevalence of Low Vision and Blindness in a Canadian Inner City. Eye, 21, 528-533. https://doi.org/10.1038/sj.eye.6702257

[23] West, S.K. (2000) Looking Forward to 20/20: A Focus on the Epidemiology of Eye Diseases. Epidemiologic Reviews, 22, 64-70.

https://doi.org/10.1093/oxfordjournals.epirev.a018025 
[24] Gilbert, C.E. and Ellwein, L.B. (2008) Refractive Error Study in Children Study Group. Prevalence and Causes of Functional Low Vision in School-Age Children: Results from Standardized Population Surveys in Asia, Africa, and Latin America. Investigative Ophthalmology \& Visual Science, 49, 877-881. https://doi.org/10.1167/iovs.07-0973

[25] Hansen, E., Flage, T. and Rosenberg, T. (1992) Visual Impairment in Nordic Children. III. Diagnosis. Acta Ophthalmologica, 70, 597-604. https://doi.org/10.1111/j.1755-3768.1992.tb02139.x

[26] Khan, S.A. (2000) A Retrospective Study of Low-Vision Cases in an Indian Tertiary Eye-Care Hospital. Indian Journal of Ophthalmology, 48, 201-207.

[27] Leat, S.J., Fryer, A. and Rumney, N.J. (1994) Outcome of Low Vision Aid Provision: The Effectiveness of a Low Vision Clinic. Optometry and Vision Science, 71, 199-206. https://doi.org/10.1097/00006324-199403000-00009

[28] Reeves, B.C., Harper, R.A. and Russell, W.B. (2004) Enhanced Low Vision Rehabilitation for People with Age Related Macular Degeneration: A Randomized Controlled Trial. British Journal of Ophthalmology, 88, 1443-1449. https://doi.org/10.1136/bjo.2003.037457

[29] Van Rens, G.H., Chmielowski, R.J. and Lemmens, W.A. (1991) Results Obtained with Low Vision Aids. A Retrospective Study. Documenta Ophthalmologica, 78, 205-210. https://doi.org/10.1007/BF00165682

[30] Temel, A. (1989) Low Vision Aids (Evaluation of 185 Patients). Ophthalmic \& Physiological Optics, 9, 327-331. https://doi.org/10.1111/j.1475-1313.1989.tb00918.x

[31] Clare, G. and Karin, V.D. (2012) When Someone Has Low Vision. Community Eye Health Journal, 25, 4. 